\title{
Gait Learning-Based Regenerative Model: a Level Set Approach
}

\author{
Muayed S. Al-Huseiny, Sasan Mahmoodi, and Mark S. Nixon \\ University of Southampton, School of Electronics and Computer Science, UK \\ mssah07r,sm3,msn(@ecs.soton.ac.uk)
}

\begin{abstract}
We propose a learning method for gait synthesis from a sequence of shapes(frames) with the ability to extrapolate to novel data. It involves the application of PCA, first to reduce the data dimensionality to certain features, and second to model corresponding features derived from the training gait cycles as a Gaussian distribution. This approach transforms a non Gaussian shape deformation problem into a Gaussian one by considering features of entire gait cycles as vectors in a Gaussian space. We show that these features which we formulate as continuous functions can be modeled by PCA. We also use this model to in-between (generate intermediate unknown) shapes in the training cycle. Furthermore, this paper demonstrates that the derived features can be used in the identification of pedestrians.
\end{abstract}

\section{Introduction}

The construction of shape models has been an area of active research. Many applications share the need for good dependable shape models which should enjoy two distinctive features: to be flexible enough to extrapolate beyond learning material, while being accurate enough to generate plausible estimates. The point distribution model proposed by Cootes et al. [2] suggesting a Gaussian model for contour deformation, has been one of the most popular models due to its simplicity. Leventon et al.[4] proposed a similar shape model which acts on signed distance functions (SDF's), a notion that avoids a strict point correspondence requirement. The common theme between these approaches is that the data they deal with, has in general a Gaussian distribution.

The issue with gait is that the distribution of the human silhouette's deformation is non Gaussian[5], and that gait is used as a sequence of shapes, such that a particular deformation has to be drawn over the whole sequence in order to produce a consistent gait cycle. Cremers[3] developed a gait model which incorporates an autoregressive (AR) system for the shape generation while preserving the time coherence of the generated shapes. This approach inherits the potential AR weakness due to its linear nature which does not match the gait features. Al-Huseiny et al.[1] used a cubic spline with the statistical shape model to in-between the training cycle, this technique lacks the ability to generalize outside the training gait cycles.

This paper proposes a new method to model shape deformations of the entire gait sequences rather than individual shapes. Considering the problem from this perspective seems appealing, since gait shapes are usually used in the context as complete cycles and not particular shapes. This can be used to generate prior shapes in a prior shapes segmentation framework, and hence clean data is used to train the model. It can also be used in classification, in which case we assume that the segmented shapes are clean.

Our proposed model is based on using PCA twice, first, to reduce the data dimensionality and extract the shapes' eigenmodes, and second to model the deformation of these eigenmodes with a Gaussian distribution. We notice that the deformation of shapes in a gait cycle along the time axis is non Gaussian. However it is assumed here that the deformation of shapes over various gait cycles at a certain time is indeed Gaussian.

So in the rest of this paper we describe our model in section 2, present the experimental outcomes in section 3 , and draw a conclusion in section 4 .

\section{Gait Regenerative Model}

In this section we describe the proposed framework to model a set of gait cycles. The boundaries of the training data consisting of $M$ gait cycles with different number of shapes per cycle, are embedded as the zero level sets of $N$ SDF's, $\mathbf{v}_{i}$ using fast marching [6], where $N$ is the total number of shapes in the training set. A mean shape is computed as $\overline{\mathbf{u}}=\frac{1}{N} \sum_{i=1}^{N} \mathbf{v}_{i}$, this shape is then subtracted from the shapes to centralize the data, and the resulting distance maps $u_{i}$ are then vectorized and augmented into the matrix $\mathbf{S}$ of shape vectors $\mathbf{u}_{i}$.

$$
\mathbf{S}_{n \times N} \equiv\left[\mathbf{u}_{1}^{1}, \ldots, \mathbf{u}_{l}^{1}, \ldots, \ldots, \mathbf{u}_{1}^{M}, \ldots, \mathbf{u}_{p}^{M}\right],
$$


the superscripts refer to the particular gait cycle, and the subscripts refer to a shape within a cycle.

$\mathbf{S}$ is then subjected to the first principal component decomposition, $\mathrm{PCA}_{\text {basis }}$, to generate a common eigenvector basis $\psi$ for the entire data set,

$$
\psi \lambda \psi^{T}=\left(\mathbf{S ~ S}^{T}\right) \times 1 / N .
$$

$\mathrm{PCA}_{\text {basis }}$ is crucial, because the projection of the shapes on the common $\psi$ produces eigenmodes $\alpha$ corresponding to appropriate shapes,

$$
\alpha_{i}=\psi^{T} \mathbf{u}_{i}
$$

Each cycle is now represented by a set of shapes' eigenmodes. The variations of these vectors we argue belong to a Gaussian distribution, and therefore a second decomposition, $\mathrm{PCA}_{\text {feature }}$ is applied to calculate the eigenvectors associated with these vectors.

The issue is that these eigenmode vectors, if considered as sampled periodic signals, are of different lengths and in different phases. A Hermite cubic spline is therefore used to represent the underlying continuous function for each vector,

$$
\alpha(t)=\operatorname{spline}(\alpha),
$$

these continuous shape eigenmodes $\alpha(t)$ are aligned and augmented as the columns of a zero-mean feature matrix $\mathbf{F}$,

$$
\mathbf{F} \equiv\left[\alpha_{1}(t), \ldots, \alpha_{M}(t)\right]_{\infty \times M}
$$

The feature covariance matrix $\mathbf{C}_{\infty \times \infty}=\mathbf{F} \mathbf{F}^{T}$, has infinite dimensions and the decomposition of its eigenvector components is not numerically tractable. A finite dimensional kernel $\mathbf{K}$ is therefore defined as

$$
\mathbf{K}_{M \times M}=\mathbf{F}^{T} \mathbf{F} .
$$

Since the vectors of $\mathbf{F}$ are continuous functions, then the components of $\mathbf{K}$ can be computed by the following integration:

$$
k_{i, j}=\int_{\tau} \alpha_{i}(t) \alpha_{j}(t) \mathrm{d} t .
$$

Now, the eigenvector decomposition of $\mathbf{K}$ gives

$$
\nu \eta \nu^{T}=\mathbf{K} .
$$

The eigenvectors $\phi(t)$, of the matrix $\mathbf{C}$ are found, [2]

$$
\phi=\mathbf{F} \nu .
$$

Equations (4-9) show that PCA can be applied to continuous functions as effectively as with discrete data.
In other words, $\mathrm{PCA}_{\text {feature }}$ places an instantaneous Gaussian model across the continuous functions at every time instant. The coefficients $\beta$ corresponding to the eigenmodes of a cycle $i$ are computed as:

$$
\beta^{i}=\phi^{T}\left(\alpha^{i}-\bar{\alpha}\right)
$$

where $\bar{\alpha}$ is the mean set of eigenmodes computed as $\bar{\alpha}=\frac{1}{M} \sum_{i}^{M} \alpha^{i}$. These computed values, $\beta^{i}$ can only be used to recall the eigenmodes $\alpha^{i}$ of the training set, a generalization into estimating novel data, $\hat{\alpha}$ means setting new values, $\hat{\beta}$ different from the computed $\beta$ 's. A set of eigenmodes $\hat{\alpha}(t)$ is then computed using:

$$
\hat{\alpha}=\phi \hat{\beta}+\bar{\alpha} \text {. }
$$

These eigenmodes $\hat{\alpha}(t)$ are then translated into an estimated cycle of vectorized gait shapes by Eq. (12),

$$
\hat{\mathbf{u}}_{t}=\psi \hat{\alpha}(t)+\overline{\mathbf{u}},
$$

where $\psi$ and $\overline{\mathbf{u}}$ are respectively the eigenvector basis and the mean shape of PCA basis.

\section{Evaluation}

We have used the training cycles from the Southampton Gait database[7]. The images are initially manually segmented and their SDF's are then generated [6].

\subsection{Novel Data Generation}

The main contribution of this work is that it consistently facilitates the manipulation of non Gaussian data in a linear fashion. That is to say, the generation of a novel cycle is achieved by adding a linear combination of the eigenvectors to the mean cycle of shapes. Hence, the model is trained using 20 gait cycles of different subjects with a varying number of frames for each cycle. Then using Eq. (11), three new cycles (shown in Fig. 1) are generated by assigning 3 different values to the first element of $\hat{\beta}$ and setting the rest to zero. Each value set to $\hat{\beta}_{1}$ produces a different set of eigenmodes $\hat{\alpha}$, which in turn using Eq. (12) generate a new cycle of gait shapes. For the sake of measurement, an $L^{2}$-norm distance $D$ is computed between the set of eigenmodes $\alpha^{i}$ for each of the training cycles and the mean set of eigenmodes $\bar{\alpha}$. This measure is intended to show the significance of the computed $\hat{\alpha}$ 's from $\bar{\alpha}$ compared to the significance of $\alpha$ 's of the training data from $\bar{\alpha}$ for the chosen $\hat{\beta}_{1}$. The distances $D$ corresponding to the chosen coefficients $\hat{\beta}_{1}$ are shown in Fig. 2. This indicates that changing one coefficient $\hat{\beta}_{1}$, produces a significantly new set of eigenmodes $\hat{\alpha}$, which gives rise to a novel gait cycle. Visually, it is easy to see from Fig. 1 the linear effect of changing $\hat{\beta}_{1}$ in producing new sequences appearing in the rows (b-d). 
(a)

AllikMS

(b) SMIMKAS

(c)

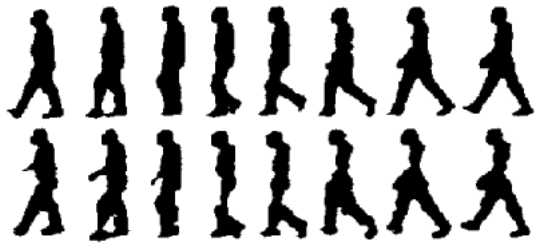

Figure 1. Row (a) is a cycle computed using the mean eigenmodes $\bar{\alpha}$ by setting $\hat{\beta}=0$. The rows (b-d) are novel cycles computed using $\hat{\beta}_{1}=$ $30, \hat{\beta}_{1}=-9 \times 10^{4}$, and $\hat{\beta}_{1}=18 \times 10^{4}$ respectively

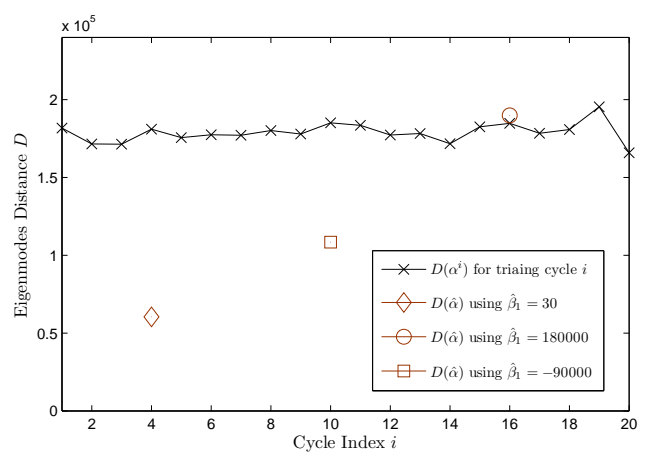

Figure 2. The distance $D$ computed for the eigenmodes $\alpha^{i}$ of the training set, and for the three novel eigenmode sets $\hat{\alpha}$ generated by setting three new distinct values to $\hat{\beta}_{1}$

\subsection{Reconstruction: Leave One Out}

We assess reconstruction by regenerating sequences of human walking subjects for our gait data. The leave one out test has proved to be a useful method to assess the reconstruction accuracy. Accordingly, for one of the training gait cycles $i$, a shape $j$ is removed each time, the remaining shapes of this cycle with the other 19 cycles together constituting the training set are used to train the model. We then reconstruct the $i^{t h}$ cycle using Eq. (11) and (12) by setting $\hat{\beta}$ to $\beta^{i}$ corresponding to the cycle $i$. The reconstructed shapes are those used to train the model as well as the one removed. This model proves robust against overfitting and learns the underlying trend of the data, which literally led to the generation of a good estimate to the missing shape. An error function $E r$ between the removed shapes and their

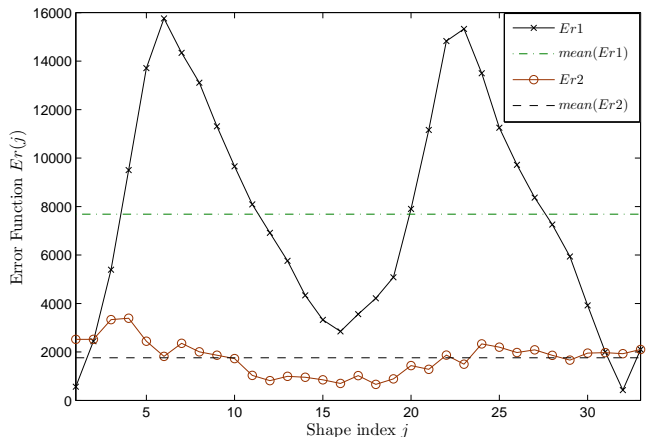

Figure 3. A comparison of the error function $E r$ in the estimated shapes, $E r 1$ computed for the shapes estimated using [1], $\operatorname{Er} 2$ computed for the shapes estimated using the proposed model

reconstructed estimates is computed as:

$\operatorname{Er}(j)=\sqrt{\operatorname{tr}\left(\left(\mathbf{v}_{r m v}(j)-\mathbf{v}_{e s t}(j)\right)^{T}\left(\mathbf{v}_{r m v}(j)-\mathbf{v}_{e s t}(j)\right)\right)}$,

where $\mathbf{v}_{r m v}$ is the removed shape and $\mathbf{v}_{e s t}$ is the estimated shape. This norm counts the number of erroneous elements in the estimate compared to the reference shape. Fig. 3 compares the error function $\operatorname{Er} 1$ computed using [1], and the error function $\operatorname{Er} 2 \mathrm{com}-$ puted using this model to reconstruct the same cycle. It is noted that the average error is four times less using the model proposed here. The reason for this is that in this model the missing shape is estimated by combining the effort of the instantaneous Gaussian contributed by PCA $_{\text {feature }}$ with the general trend of the data captured by the cubic spline, while in [1], the estimation is based purely on the estimation of general trend of the data.

\subsection{Pedestrian Identification}

The shape eigenmodes generated by our model can be employed in the identification of pedestrians based on their gait by using the following theorem.

Theorem: In the model proposed here:

$$
d=\int_{\tau}\left\|\mathbf{V}^{1}-\mathbf{V}^{2}\right\|^{2} \mathrm{~d} t=\sum_{i} \int_{\tau}\left\|\alpha_{i}^{1}-\alpha_{i}^{2}\right\|^{2} \mathrm{~d} t,
$$

where $\mathbf{V}^{1}$ and $\mathbf{V}^{2}$ are the vectorized SDF's of the gait cycles for two subjects and $\alpha^{1}$ and $\alpha^{2}$ are their corresponding eigenmodes.

Proof: Let $\mathbf{V}^{1}$ and $\mathbf{V}^{2}$ be the gait cycles for two subjects with $R$ shapes each, such that:

$$
\mathbf{V}^{k}=\overline{\mathbf{v}}^{k}+\sum_{i=1}^{R} \psi_{i}^{k} \alpha_{i}^{k}(t)
$$




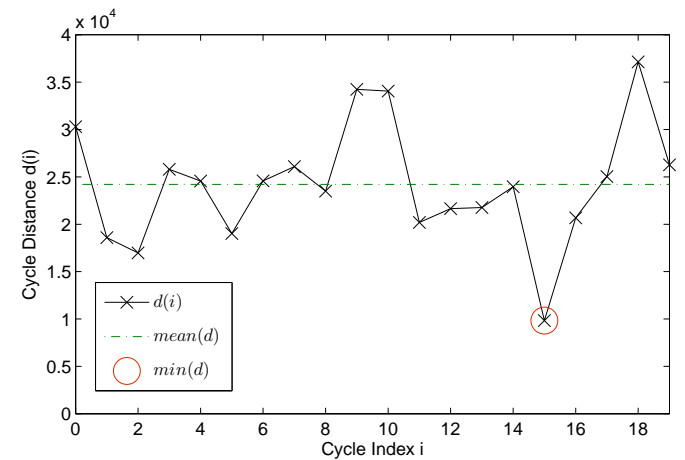

Figure 4. The correct cycle has the least distance $d$ to the test cycle

where $k=1,2$; then the distance $d$, between the two cycles is computed by:

$$
\begin{aligned}
d & =\int_{\tau}\left\|\mathbf{V}^{1}-\mathbf{V}^{2}\right\|^{2} \mathrm{~d} t \\
& =\int_{\tau}\left\|\overline{\mathbf{v}}^{1}+\sum_{i} \psi_{i}^{1} \alpha_{i}^{1}(t)-\overrightarrow{\mathbf{v}}^{2}-\sum_{j} \psi_{j}^{2} \alpha_{j}^{2}(t)\right\|^{2} \mathrm{~d} t .(17)
\end{aligned}
$$

Since all the shapes in the proposed model are represented using a common mean shape $\overline{\mathbf{u}}$ and eigenvectors $\psi$, then $\overline{\mathbf{v}}^{1}=\overline{\mathbf{v}}^{2}=\overline{\mathbf{u}}$, and $\psi^{1}=\psi^{2}=\psi$ and hence,

$d=\int_{\tau}\left[\sum_{i} \psi_{i} \alpha_{i}^{1}-\sum_{j} \psi_{j} \alpha_{j}^{2}\right]^{T}\left[\sum_{i} \psi_{i} \alpha_{i}^{1}-\sum_{j} \psi_{j} \alpha_{j}^{2}\right] \mathrm{d} t$

and since $\psi_{i}^{T} \psi_{j}=\delta_{i j}$, where $\delta_{i j}$ is the Kronecker delta,

$d=\int \sum_{\tau} \alpha^{1^{2}}-2 \alpha^{1} \alpha^{2}+\alpha^{2}=\sum_{i} \int_{\tau}\left\|\alpha_{i}^{1}-\alpha_{i}^{2}\right\|^{2} \mathrm{~d} t$

This distance $d$ is used in the identification to measure the closest training cycle to an unknown cycle. So, for one of the subjects (chosen arbitrarily) in the training set, a new unknown cycle (not in the training set) is used as the test cycle, the distance $d$ is computed between the eigenmodes of this test cycle and the eigenmodes of the known 20 cycles of the training set. Fig. 4 shows the outcomes, in which, it is seen that the correct cycle is selected by calculating the least distance $(d)$.

\section{Conclusions}

In this paper we proposed a gait model that transforms the problem of non Gaussian shape deformation, into a Gaussian one by considering entire gait cycles as training data points and modeling them using PCA. We have applied this model to human gait analysis and synthesis, though it could be applied elsewhere. This approach preserves, and meanwhile exploits the time coherence of the shapes in the gait, which is important in applications like tracking and prior shape based segmentation with occluded images. The pioneering employment of PCA in handling continuous functions made it possible to align the gait cycles which made the case for accurate automatic shape correspondence. The introduction of PCA over corresponding shapes resulted in producing the entire novel cycles with valid shapes by changing the model coefficients which is the main practical outcome of this model.

The proposed model was also used successfully in reconstructing the training data. Furthermore it proved more robust in the reconstruction of in-between shapes compared to the previous approaches. These results presented in this paper, demonstrate that the proposed method enjoys the flexibility of the statistical methods with the accuracy of deterministic techniques.

The model proposed here, has showed and proved that the statistical shape eigenmodes can be used in pedestrian identification, which can be extended into a gait biometric by applying this model to a large database, without the need for computing complex shape descriptors, such as the statistical moments in order to provide the common basis for measurement.

Acknowledgment: This work is partially supported by PASCAL2.

\section{References}

[1] M. S. Al-Huseiny, S. Mahmoodi, and M. S. Nixon. Level set gait analysis for synthesis and reconstruction. In ISVC, pages 377-386, 2009.

[2] T. F. Cootes, C. J. Taylor, D. H. Cooper, and J. Graham. Active shape models-their training and application. Comput. Vis. Image Underst., 61(1):38-59, 1995.

[3] D. Cremers. Dynamical statistical shape priors for level set-based tracking. IEEE TPAMI, 2(8):1262-1273, 2006.

[4] M. E. Leventon, W. E. L. Grimson, and O. Faugeras. Statistical shape influence in geodesic active contours. In CVPR, pages 316-323, 2000.

[5] S. Mahmoodi. Shape-based active contours for fast video segmentation. IEEE Sig. Proc. Lett., 16(10):857-860, 2009.

[6] J. Sethian. A fast marching level set method for monotonically advancing fronts. Proc. Natl. Acad. Sci. USA, 93(4):1591-1595, 1996.

[7] J. Shutler, M. Grant, M. Nixon, and J. Carter. On a large sequence-based human gait database. In Intl. Conf. on Recent Advances Soft Computation, pages 66-71, 2002. 